\title{
Artificial Neural Network in Drug Delivery and Pharmaceutical Research
}

\author{
Vijaykumar Sutariya ${ }^{\mathrm{a},{ }^{*},}$, Anastasia Groshev ${ }^{\mathrm{a}}$, Prabodh Sadana ${ }^{\mathrm{b}}$, Deepak Bhatia ${ }^{\mathrm{b}}$ and Yashwant \\ Pathak $^{\mathrm{a}}$ \\ ${ }^{a}$ Department of Pharmaceutical Sciences, USF College of Pharmacy, University of South Florida, Tampa, FL 33612 \\ ${ }^{b}$ Department of Pharmaceutical Sciences, College of Pharmacy, Northeast Ohio Medical University, Rootstown, OH \\ 44272
}

\begin{abstract}
Artificial neural networks (ANNs) technology models the pattern recognition capabilities of the neural networks of the brain. Similarly to a single neuron in the brain, artificial neuron unit receives inputs from many external sources, processes them, and makes decisions. Interestingly, ANN simulates the biological nervous system and draws on analogues of adaptive biological neurons. ANNs do not require rigidly structured experimental designs and can map functions using historical or incomplete data, which makes them a powerful tool for simulation of various non-linear systems.ANNs have many applications in various fields, including engineering, psychology, medicinal chemistry and pharmaceutical research. Because of their capacity for making predictions, pattern recognition, and modeling, ANNs have been very useful in many aspects of pharmaceutical research including modeling of the brain neural network, analytical data analysis, drug modeling, protein structure and function, dosage optimization and manufacturing, pharmacokinetics and pharmacodynamics modeling, and in vitro in vivo correlations. This review discusses the applications of ANNs in drug delivery and pharmacological research.
\end{abstract}

Keywords: Artificial neural networks, ANNs, drug discovery, non-linear systems, pharmacokinetics, pharmacodynamics.

\section{INTRODUCTION}

In the past decade, neural networks have received a great deal of attention among scientists and engineers and they are being touted as one of the greatest computational tools ever developed. Much of this excitement is due to the ability of neural networks to emulate the brain's ability to learn by example. This network makes decision and draws conclusionseven when presented with incomplete information. Moreover, at some primitive level, neural network imitates brain's creative process in adapting to a novel situation [1]. It is a very good statistical tool for many numeric as well as nonnumeric calculations. Specifically, ANNs are known to be a powerful tool to simulate various non-linear systems and have been applied to numerous problems of considerable complexity in many fields, including engineering [1], psychology, medicinal chemistry $[2,3]$, diagnostics $[4,5]$, and pharmaceutical research [6].

\section{ARTIFICIAL NEURAL NETWORKS MODELING}

As biologically inspired computational model, ANN is capable of simulating neurological processing ability of the human brain. Average human brain contains about 100 billions of neurons with each neuron being connected with 1000-10,000 connections to others. A single neuron consists

*Address correspondence to this author at the Department of Pharmaceutical Sciences, University of South Florida College of Pharmacy, Tampa, Florida, 33612-4749, Tel: 813-974-1401; Fax: 813-974-9890;

E-mail: vsutariy@health.usf.edu of three major parts - dendrites (fine branched out threads) carrying signals into the cell, the cell body receiving and processing the information, and the axon (a single longer extension) (Fig. 1). The axon carries the signal away and relays it to the dendrites of the next neuron or receptor of a target cell. The signals are conducted in all-or-none fashion through the cells. All the connections in the brain enable it to learn, recognize patterns, and predict outcomes.Similarly to the brain, ANN is composed of numerous processing units $(\mathrm{PE})$, artificial neurons. The connections among all the units vary in strength, which is defined by coefficients or weights. The ANN mimics working of human brain and potentially fulfills the cherished dream of scientists to develop machines that can think like human beings.ANNs simulate learning and generalization behavior of the human brain through data modeling and pattern recognition for complex multidimensional problems. A significant difference between an ANN model and a statistical model is that the ANN can generalize the relationship between independent and dependent variables without a specific mathematical function. Thus, an ANN works well for solving nonlinear problems of multivariate and multiresponse systems such as space analysis in quantitative structure-activity relationships in pharmacokinetic studies [7] and structure prediction in drug development [8].

There are many types of neural networks with new ones being continually invented; however, all ANNscan be characterized by their transfer functions of their processing units (PE), the learning rules, and by the connections formulas. PE, building component of $\mathrm{ANN}$, receives many signals as weighted process variables from the response of 


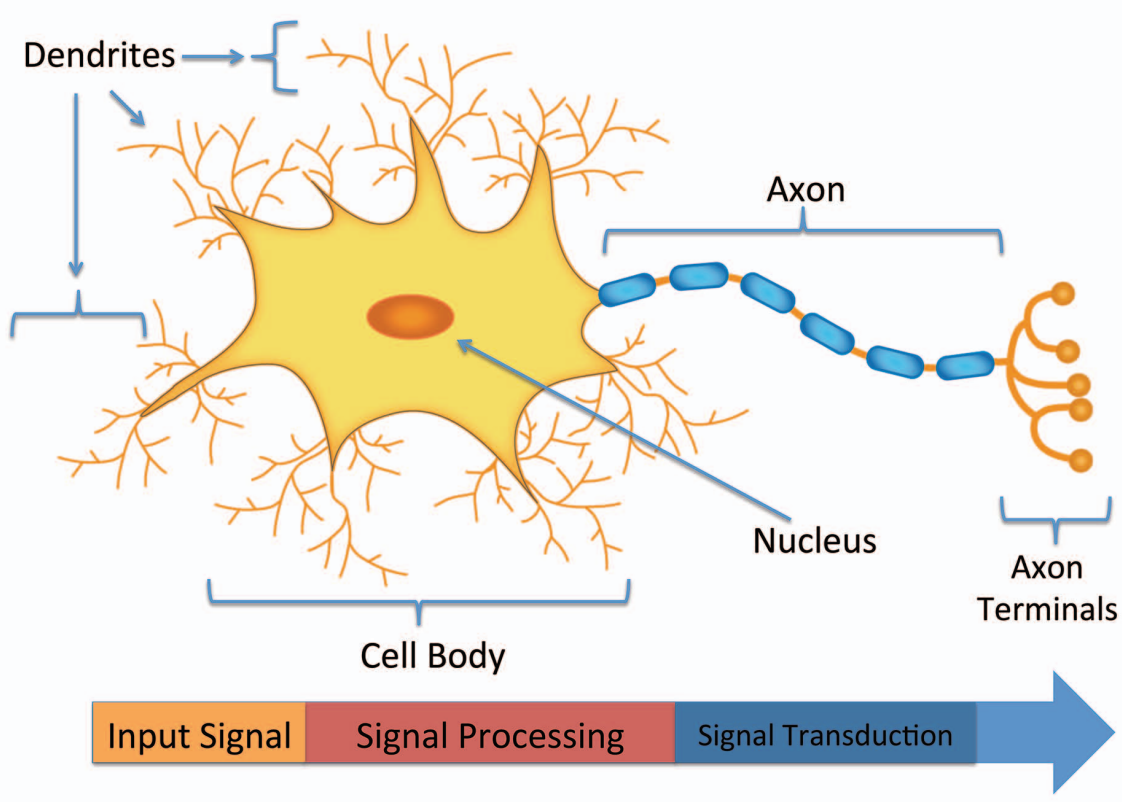

(A)

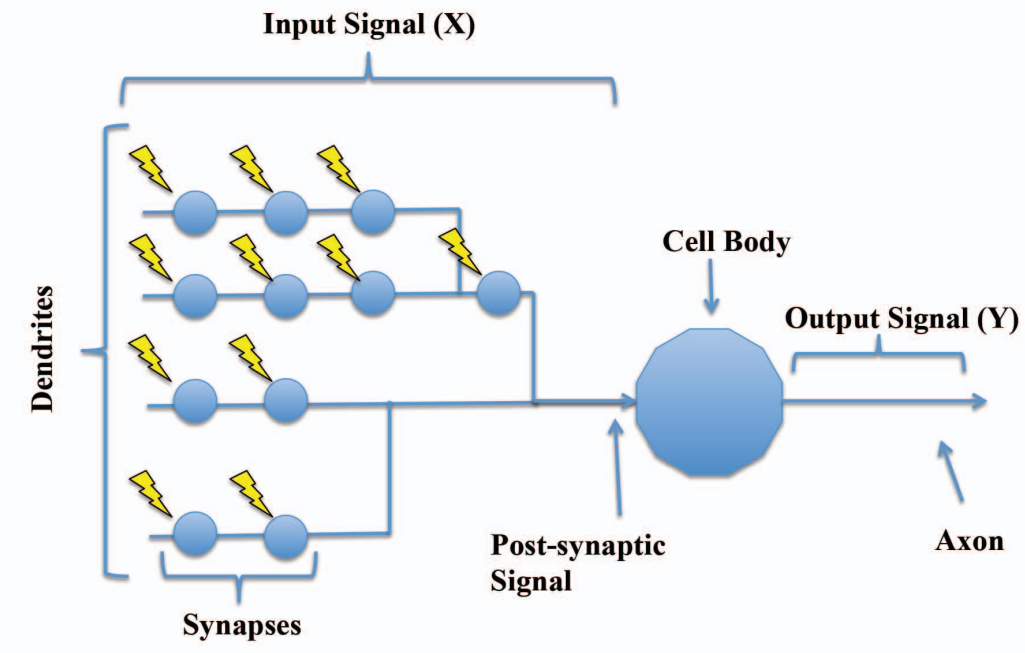

(B)

Fig. (1). Conceptual structure of a biological neuron. (A) Schematic representing structure of biological neuron. Branched out dendrites receive the signal. The cell body processes the signal and relays it to the next target via axon, the elongated extension. (B) The input signals, represented by lightning bolts stimulate the dendrites in the synapses. The signals are relayed to the cell body, where they are processed and travel onto the axon as a single output signal.

other units [9]. The most commonly applied ANN layout is forward propagating network trained by error backpropagation developed by Rumehart et al. [10]. The forward propagation network consists of input layer, one or more hidden layers and one output layer (Fig. 2) [11]. The input layer provides data from the external source. The mapping of the input data occurs by neural network hidden layers, then the final representative signal is generated by the output layer $[10,12]$. The ability of neural networks to classify information depends on hidden layers, which are fully connected by the synapses to the neighboring layers.

In each hidden layer and output layer, the processing unit sums up its input from previous layer by the sigmoidal function to compute the output to the following layer according to the following equations (1) and (2) [13].

$y_{q}=\sum w_{p q} x_{p}$ 


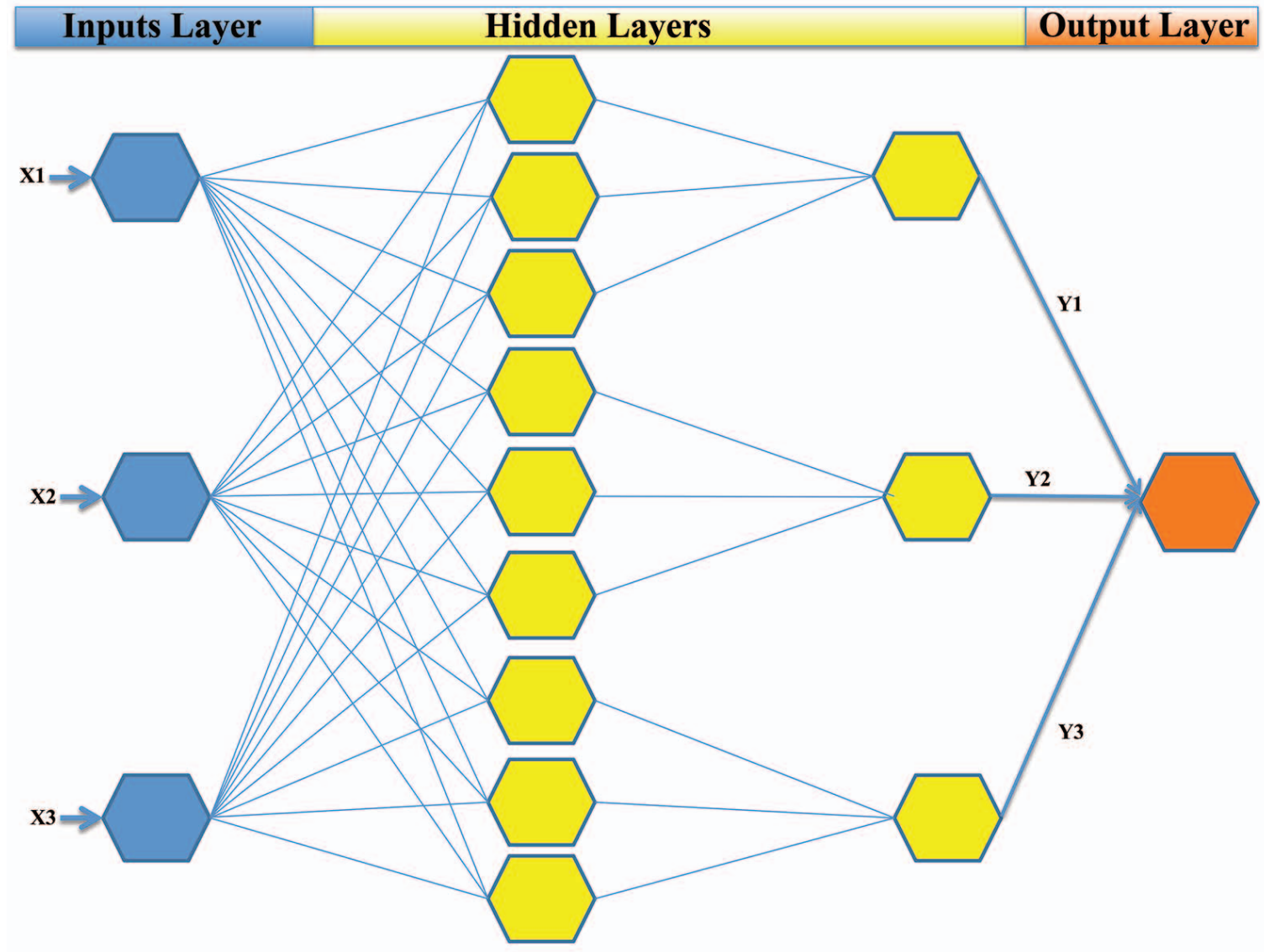

Fig. (2). A schematic of four-layered artificial network. Input layer units (in blue) receive input signals $\left(\mathrm{x}_{1}, \mathrm{x}_{2}, \mathrm{x}_{3}\right)$ and transfer the signal to the hidden layers via weighted connections. Output layer receives the signals and provides the representative output signal.

$$
f\left(y_{q}\right)=\frac{1}{1+\exp \left(-\alpha y_{q}\right)}
$$

Where, $\mathrm{w}_{\mathrm{pq}}$ is the strength of the connections between unit $\mathrm{q}$ in the current layer to unit $p$ in the previous layer, $x_{p}$ is the output value from the previous layer, $\mathrm{f}\left(\mathrm{y}_{\mathrm{q}}\right)$ is conducted to the following layer as an output value, and $\alpha$ is a parameter relating to the shape of the sigmoidal function. The advantage of this function is that it can accommodate a large signal without saturation while allowing small signals to pass without excessive attenuation. Nonlinearity of the sigmoidal function is strengthened with an increase in $\alpha$. The ANN learns an approximate nonlinear relationship by a procedure called training, which is the search process for the optimized set of weight values to minimize the squared error between the estimation and experimental data of units in the output layer. Most commonly used methods is back-propagation method, whichrequires three simple steps-network design, learning or training, and usage [11]. In the network design stage the number of connections and layers is selected based on the type of application. Then, the training stage requires of selection of training set of data and remodeling of the network to minimize the error. And lastly, following the training ANN is suitable to use.

Network design. Number of hidden layers is essential to the purpose and function of an ANN as it influences the number of connections in the network and, thus, its performance. A very common approach to select the optimal number of hidden nodes is by trial and error method using the training rules as guidance; however, the upper limit of number of hidden nodes on an ANN model can be determined using the following equation (3) [14].

$$
n_{s}=\beta\left\{n_{h}\left(n_{i}+1\right)+n_{o}\left(n_{h}+1\right)\right\}
$$

Where, $\mathrm{N}_{\text {hidden }}$ is the number of hidden nodes; $\mathrm{N}_{\text {trn }}$ is the number of training sample; $R$ is a constant with values ranging from 5 to $10, N_{\text {inp }}$ is the number of inputs and $N_{\text {out }}$ is the number of outputs. The final number of process variables and response units depends on the type of the problem and is determined by the data for the analysis. After finalizing the number of layers and neurons in each layer ANN is ready for training.

Training. The process of training involves a search for the most optimal network state by adjustments of the weights of the connections between PEs. The weights are adjusted, based on the training set of data, until the error is minimized. At some point the network may become stuck in local minima, where the error is lower; however, less than optimal confirmation is achieved. The momentum can aid the network to continue sampling confirmations making training is a long iterative process.Certain empirical techniques have been reported to improve the convergence of ANNs in the global minima [15]. Kalman filter algorithm [16, 17] may help to reduce the amount of iterative trainingand prevent the ANN getting stuck in local minimaby using the simulated 


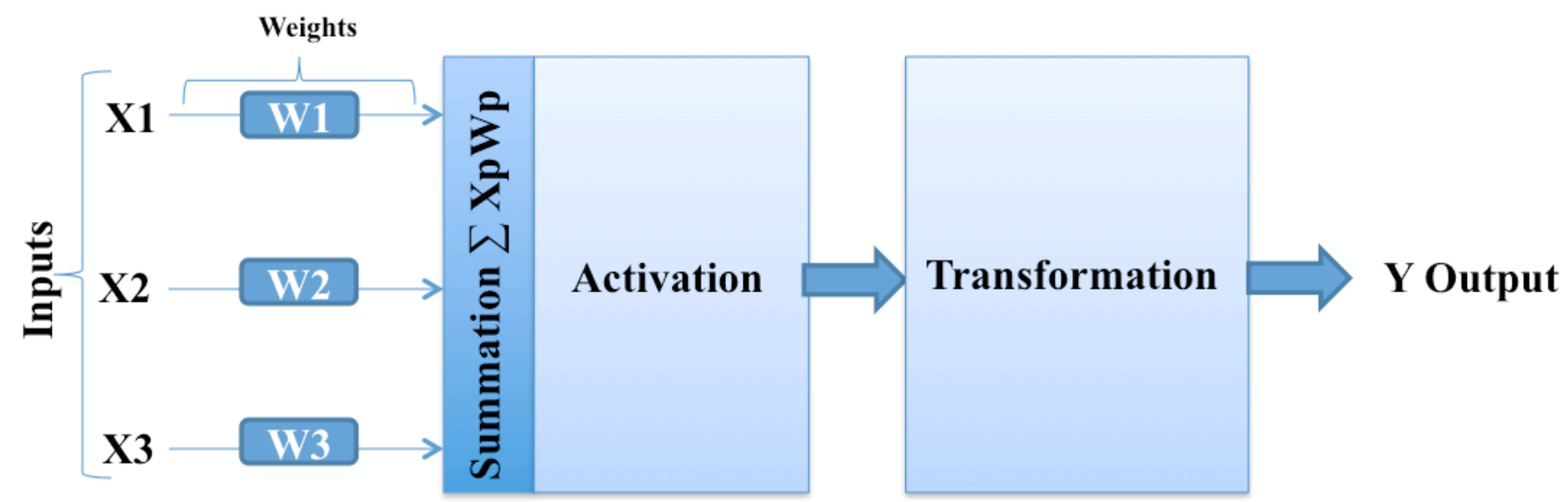

Fig. (3). A common design of a node in an artificial neural network. The input signal travels to the unit though weighted connection. Multiple signals are summated, processed, and transformed based on the specific function. An output signal is relayed to the following nodes in the network.

annealing technique where the global minima can be more specified making the training process quicker and more efficient [18-20].

The amount of training is important because undertrained ANNs yields larger errors in the output signal and overtrained ANNs lose the ability to generalize and recognize patterns. Equation (4) relating to the number of units in the input layer, the hidden layer and the optimal output layer may be used forreasonable prediction of number of training pairs [21]:

$$
A I C=n_{s} \mathrm{x} \ln (S S)+2 \mathrm{x} r
$$

Where, $n_{h}$ is the number of hidden units, $n_{i}$ is the number of input units, $n_{0}$ is the number of output units, and $n_{s}$ is the number of training data pairs. The constant $\beta$ is the parameter that relates to the degree of over determination.

Further, a cross-validation technique such as a "leaveone-out (LOO) method" may be applied to ensure the optimality of an ANN structure [22-24]. One data pair is systematically removed from the training data set, and the ANN is then trained by using the reduced data set. Akaike's information criterion (AIC) can be applied to evaluate the optimality of ANN (Equation (6)) [25]:

$$
N_{\text {hidden }}=\frac{N_{\text {tm }}}{\left[R+\left(N_{\text {inp }}+N_{\text {out }}\right)\right]}
$$

Where, $n_{s}$ is the number of data pairs, $n_{w}$ is the number of weights in the $\mathrm{ANN}$, and $\mathrm{SS}$ is the residual sum of squares between observed and predicted response variables [26].

The learning through weight adjustment can be supervised or unsupervised. The network is repeatedly presented with an input pattern and a desired output response in supervised learning. The training process terminates when error goal is near zero and neural network produces correct response for given input patterns. In unsupervised learning no desired response is available to guide system and the learning is through input pattern alone. The neural network system itself then decides features to be used for grouping input data and this process is called self-organization or adaptation. The speed of learning is actually the rate of convergence between the current solution and the global minimum. Momentum helps the network to overcome obstacles (local minima) in the error surface and settle down at or near the global minimum. The most common training algorithm is based on the Delta rule,according to which, each of the training iterations (frequently referred to as "epoch") are described by the following general equation:

New weight change $=$ learning rule $*$ Error + Momentum + Last weight change

The feed forward step starts with presentation of process variable pattern and continues through activation level to propagate through hidden layers. The processing unit sums the input and applies sigmoidal function in the hidden layer to compute its response as shown in Fig. (3). In feed-back step, error values are calculated for all processing units and weight changes are calculated for all interconnections. The calculations start at output layer and progress backward through the network to input layer. Thus each neuron has one additional weight as an input that allows an additional degree of freedom when trying to minimize training error.

In the training process, it may become necessary to change the number of units in the hidden layers, especially in multilayer networks where overfitting may occur. Pukrittayakamee et al. described two types of overfitting that may occur during the training process when both function and its derivatives and recommended pruning or adjusting the number of PEs in the network to overcome this issue [27].

Based on the design and purpose of the ANN, the training may reach termination in the state of minimal error or continue evolving and learning though interactions with datasets. If the data is noisy, overtraining of the ANN may be of a concern as it may lose the ability to generalize and recognize the patterns. However, evolving ANNs are often used for modeling brain activity and artificial intelligence [28], space perception [29], and analysis of data requiring large inputs and large outputs [30] among other applications.

\section{APPLICATION OF ANNS MODELING IN DRUG DELIVERY AND PHARMACEUTICAL RESEARCH}

The potential applications of ANN methodology in the pharmaceutical sciences are broad as ANNs capabilities can 
be summarized bymodeling, pattern recognitionand prediction. Thus, applications of ANNs includedrug modeling, dosage design, protein structure and function prediction, pharmacokinetics and pharmacodynamics modeling, as well as, interpretation of analytical data, and in vitro/in vivo correlations.

\subsection{ANNs Applications Analytical Data Analysis and Structure Retention Relationship (SRR) Methodology in Pharmacological Research}

As ANNs can recognize patterns from complex sets of analytical data, they become very useful in data analysis of pharmacological research due to their ability to recognize even non-linear relationships from noisy data. For instance, ANNs can be applied in analysis of spectral data of multicomponent samples such as mixtures forquantification of the concentrations in the mixture using the whole spectrum in the identification process [31-33]. Hasani et al. used ANNs for detection and calibration of amino acids with similar structures and spectrums such as tryptophan, tyrosine, and histidine [32]. Moreover, ANNs are capable of assisting in determining of concentrations of a chiral sample and enantiomeric excess in a single spectrophotometric measurement due to their ability to identify non-linear relationships [34]. For instance, ranitidine hydrochloride, one of the most commonly prescribed antihistamines, exists in two forms, Form 1 and 2. Application of ANNs in development of method of quantification of ranitidine-HCL using diffuse reflectance IR spectral data and X-ray diffraction allows for sensitive and quick identification of Form 1 concentration in a multi-component tablet without extraction of active ingredient and Form 1 [35-37]. Usefulness of ANNs in analysis of peptide MS/MS spectral data has been also demonstrated. The constructed ANN was used to analyze the data generated by Sequest, a widely used protein identification program. ANN was demonstrated to classify automatically as either "good" or "bad" the peptide MS/MS spectra otherwise classified manually. An appropriately trained ANN proves to be a highthroughput tool facilitating examination of Sequest's results and authors recommended a routine use of this approach in handling large MS/MS data sets [38].

ANNs can also be used as the basis of computer-assisted optimization method for selection of optimal gradient conditions for anion separations in chromatography [39-41]. The ANNs with 1-10-9 architecture has been found to be rapid and accurate in predicting retention times for anions in linear gradient elution ion chromatography with hydroxide eluents. Tham et al. applied ANNs in quantitative structuregradient elution retention relationship of the compounds such as phenyl thiocarbamyl amino acids derivatives with estimation of the chromatographic retention times [42]. This study found the training set RMS error to be 1.773 and the testing set RMS error- 0.8377 . Based on the RMS errors of the training and testing sets and high correlation of predicted versus experimental values $(\mathrm{R}>0.97)$, it is clear that the correlation between the structure and the chromatographic separation can be predicted by ANNs. Similarly, ANNs can assist in HPLC optimization by finding correlation of the chromatographic behavior of solutes (capacity factors) with mobile phase composition and $\mathrm{pH}$ to predict retention times
[43], the separation as a function of simultaneous change in $\mathrm{pH}$ and solvent strength [44, 45], and hydrophobicity coefficients for the prediction of peptide elution profiles [46]. ANNmodel have used in successful prediction of retention values of unanalyzed molecular in studies done by Agatonovic-Kustrin by determining the correlations of chromatograms retention times with mobile phase composition and $\mathrm{pH}$, and with physical chemical properties of amiloride, hydrochloride and methyldopa [47]. Recently, Chen et al. have used ANNs in combination with generic algorithms (GA) for method optimization of detection of two similar biophenols blood levels even at trace amounts and small sample volume [48]. ANNs can serve as a great tool in optimization stage of the method development to help save time and cost during this stage of research. Further, ANNs may help to detect relationships in analysis of challenging sets of data such as characterization of mixtures.

\subsection{Preformulation}

ANN model has been used in the design and optimization of preformulation to determine the physicochemical propertiesof amorphous polymers [49]. A study by Ebubeet. al. indicated that the ANN model accurately predicted the water-uptake, glass transition temperatures and viscosities of different hydrophilic polymers and their physical blends with a low prediction error $(0-8 \%)$. It demonstrated the potential of the ANNs as a preformulation tool as trained ANNs model enables prediction of the relationships between the compositions of polymer blend and the water uptake profiles, the relationships between composition of polymers blends and viscosity of polymers solutions, and the relationship between moisture content of polymers and their glass transition temperatures.

In the phase design, it may be important to consider the effect of active pharmaceutical ingredients (API) on the tablet itself, especially if multiple APIs are present. Using ANNs,Onukiet. al. were able to test 14 APIsafter optimizing the formulation base using self-organizing maps in order to expand the fast-release tablet database and study the effect of API on the tablet properties [50]. Moreover ANNs also have been successfully used in design of stable formulations for multiple active components, such as rifampicin and isoniazid microemulsions [51]. ANNs are certainly very useful in the preformulation design and would help reduce the cost and length of preformulation study.

\subsection{Optimization of Pharmaceutical Formulations}

The prediction of pharmaceutical responses based on the polynomial equation and response surface methodology (RSM) has been widely used in formulation optimization but may be limited to low volumes, resulting in the poor estimation of optimal formulations. In order to overcome these shortcomings, a multi-objective simultaneous optimization technique incorporating an ANN has been developed [52, 53]. For example, the reliability of ANNs in optimizing controlled release capsules and ketoprofen hydrogel ointment has been demonstrated by Hussain et al [54]. A trained ANN model has been successfully employed to predict release profile and optimize formulation of various drug formulations such as aspirin extended release tablets $[55,56]$, diclofenac sodium sustained release matrix tablets 
[57], salbutamol sulfate osmotic pump tablets [58], transdermal ketoprofen hydrogel [59], and nimodipine floating tablet formulation [60].

Takayama et al. applied an ANN model to optimize controlled release theophylline tablets prepared with the mixture of hydroxypropylmethyl cellulose with lactose and cornstarch [25]. The plasma concentration profiles were simulated based on the pharmacokinetic parameters of theophylline. The results predicted by the trained ANN model agreed well with the observed values. Chen et al. has also used ANN and pharmacokinetic simulations in the design of controlled-release formulation [61]. Three out of the four predicted formulations showed very good agreement between the ANN predicted and the observed in vitro release profiles based on difference factor. Vaithiyalingam et al. used the ANN to model the effect of process and formulation variables, coating weight gain, duration of curing, and plasticizer concentration on in vitro release profile of verapamil $\mathrm{HCl}$ from multi-particulate beads formulated with a novel aqueous-based pseudolatex dispersion [62]. The observed drug release data of the optimized formulations was close to the predicted release pattern, based on the ANN model. Recently, Atkas et al. have utilized ANNs in developing a carvedilol controlled release tablets, where the formulation resisted the $\mathrm{pH}$-dependent solubility of carvedilol [63]. No significant difference between ANNderived predictions and experimental values were observed during this study. Similar findings of ANNs application in formulation optimization have been reported by Patel et. al., in their study ANN was used to design low and $\mathrm{pH}$ dependent tablet formulation [64]. Optimization process variables included drug amount, osmotic pressure of promoting agent rage, polymer content and weight of the coating, demonstrating that ANNs may be valuable in optimization of the formulations.

\subsection{In Vitro In Vivo Correlations}

The in vitro in vivo correlations (IVIVC) are of great interest for pharmaceutical industry to avoid bioequivalence studies that are predicted to produce negative results. ANNs applied to IVIVC have the potential to be a reliable predictive tool that overcomes some of the limitations associated with classical regression methods such as lack of pattern recognition powers analyzing multivariable data sets with high degree of variation. ANN configurations are very useful in prediction of IVIVC from different formulations of same product [65]. ANNs can also be applied in quantitative structure-pharmacokinetic relationship (QSPR) of betablockers using ANNs demonstrating that ANNs are capable of prediction in vivo results from in vitro experiments [66]. IVIVC has been applied in prediction of absorption of salbutamol in the lungs in healthy and asthmatic volunteers based on published in vivo data $[67,68]$. The drug concentrations were measured in urine and compared to the lung function. The study concluded that ANNs are useful in predicting in vivo response based on in vitro data based on the squared correlations between predicted and experimental values of 0.83 and 0.84 for urine and lung function data.Recent studies by Paixãoet. al. predicting drug clearance by hepatocytes based on in vitro data (train $r=0.953$ and test $\mathrm{r}=0.804$ ) show that ANNs may be a valuable tool in prediction of metabolic clearance of new drugs [69].

\subsection{Quantitative Structure-Activity Relationships (QSAR) and Quantitative Structure-Property Relatio- nship (QSPR)}

QSAR correlate structure or property descriptors of compounds with chemical or biological activities and increasing number of neural network models are currently published for predicting various physicochemical properties from the molecular structures [70-73]. All QSAR studies are based on the fundamental concept of interdependence of biological activities on physicochemical parameters. The relationships of structure and function with the physicochemical descriptors and topological parameters can be determined by computational methods. For instance, ANNs have been used by Grobburu and colleagues to predict the quantitative structure QSPR of beta adrenoreceptor antagonists in humans [74]. In the study, ANNs with congeneric series of ten beta-blockers having well established critical pharmacokinetic parameters was constructed and tested for its ability to predict the pharmacokinetic parameters from the octanol/water partition coefficient, the $\mathrm{pK}_{\mathrm{a}}$, or the fraction bound to plasma proteins. Neural networks predicted values showed better agreement with the experimental values than those predicted by multiple regression techniques (average difference $=47 \%$ ). Comparison studies of the predictive performance of a mechanistic model with an empirical ANN model in calculating the relationship between the tissue distribution and the lipophilicity of a homologous series of 5-n-alkyl-5ethyl barbituric acids in the rat have been published and demonstrated the superiority of ANNs in this application [75]. The mean prediction error (ME) of the mechanistic model was $18 \%$ (range, 20 to $57 \%$ ), indicating a tendency for overprediction; the mean squared prediction error (MSE) is $32 \%$ (range, 6 to $104 \%$ ).

On other hand, ANN model had almost no bias: the ME was $2 \%$ (range, 36 to 64 ) and had greater precision than the mechanistic model, MSE $18 \%$ (range, 4 to $70 \%$ ). ANNs have also been applied in prediction of antimicrobial activities of quinolone derivatives and detection of their minimum inhibitory concentrations using a topological method based on chemical structure of quinolone derivatives [76]. Morerecently, an improved prediction model of antimicrobial activity has been published by Torrent et. al. allowing for projection of antimicrobial potency accounting even for factors such as protein aggregation [77]. ANN also has been utilized in the development of four-parameter counter propagation (CP) QSAR model of Dunaliellatertiolecta in predicting toxicity for freshwater alga Pseudokircheneriellasubcapitata [78] and in screening of antibacterial activity of 3-hydroxypyridine-4-one derivatives [79].

Further, ANN has been useful in QSAR study of antitumor activity of acridinone derivatives [80]. Correlation of predicted values to the experimental data was 0.9484; moreover, the developed model allowed also to identify the important factors contributing to the antitumor activity such as lipophilicity. Thus, ANNs are not only useful in prediction QSARs but also in identifying the role of the 
specific factors relevant to the activity of interest. This has been demonstrated in the earlier published study by Uesawa and colleagues in their QSAR analysis of tumor-specificity of 1,2,3,4-tetrahydroisoquinoline derivatives, where ANN allowed for identification of water-accessible surface area and quantum chemical descriptors as factors in estimating tumor specificity of compounds [72].

Calculation of certain physicochemical properties before synthesizing or purchasing screening library would be especially valuable drug discovery phase. Lipophilicity and water solubility are properties related to the octanol-water partition coefficient, which can be used as rough early ADME screens to reject probable development failures as early as possible. Clark and co-workers used a data set containing 1085 compounds for developing a neural network model for octanolpartition coefficient prediction from the results of semi empirical AM1 calculations [81] and Eros et al. developed neural network (fitting and prediction errors were $\mathrm{s}=0.48$ and $\mathrm{s}=0.72$ respectively)from database of 625 molecules, $98 \%$ of which are registered API showing high structural diversity [82]. Similarly, more recent studies based on QSPR ANN model predicted octanol-water partition coefficients for 209 chlorinated trans-azobenzene derivatives, contaminants in herbicides [83]. QSPR ANN model was also used by Noorizadeh et al. to calculate the polar surface area of 32 drug molecules [84]. Application of ANNs in prediction of API solubility has been demonstrated by Louis and colleagues in comparison study of MLR, ANN, and SVM methods with ANN yielding the best prediction data set [85]. Squared correlation coefficient values were $0.814,0.823$, and 0.835 for MLR, ANN, and SVM respectively.

ANNs can also be very useful in predicting chemical properties of compounds. Several research groups have modeled the normal boiling point of hydrocarbons. Predictive neural network models have been published for alkanes [86, 87], alkenes [88] and for diverse hydrocarbons [89]. As expected, the models typically show good fitting and prediction statistics with less than ten simple descriptors. Goll and Jursalso applied artificial neural network to predict the vapor pressures of hydrocarbons and halohydrocarbons from molecular structure [90]. The neural network model with 7-3-1 architecture predicted the test set with a root mean square error of 0.209 ( $\mathrm{n}=52)$. Recently, Piliszek and colleagues utilized property parameterization model (RM1) and density functional theory (DFT) followed by ANN analysis in prediction of subcooled vapor pressures and classification of 399 polychlorinated trans-azoxybenezenes [91]. Yaffe et al. modeled Henry's law constant using both fuzzy ARTMAP and feed forward neural network, the heterogeneous data set $(n=495)$ included compounds with oxygen, sulphur and nitrogen containing functional groups and halogens [92]. The log Hvalues ranged from 26.72 to 2.87 and topological descriptors were used as input parameters. The average absolute errors for the test set of 74 members were 0.13 and $0.27 \log$ Hunits for fuzzy ARTMAP and the feed forward network, respectively. Liu et al. used five topological indices as input descriptors for the neural network with 5-5-3 architecture in predicting the refractive index, density and boiling point for alkenes and the training set contained 49 members [88]. Standard error for refractive index was found to be $0.13 \%$ and $0.4 \%$ for density using a test set of 16 alkenes.

Sild and Karelson developed ANN models for predicting dielectric constant and Kirkwood function using a data set of 155 organic liquids with extensive structural diversity and a range of 1.87-46.5 for the dielectric constant [93]. Separate models with 5-5-1 configuration were developed for both dielectric constant and the Kirkwood function. The average prediction error for the dielectric constant was $27.0 \%$ and for the Kirkwood function $4.1 \%$. Tettech et al. have also developed a radial basis forward neural network for simultaneous prediction of flash point and boiling point [94]. The database contained 400 organic compounds with flash points between $-60{ }^{\circ} \mathrm{C}$ and $200{ }^{\circ} \mathrm{C}$. The average absolute error for the test set in flash point prediction was $11.9^{\circ} \mathrm{C}$ with a 26-36-2 configuration. Suzuki et al. developed an ANN model for predicting liquid viscosity at a standard temperature of $20^{\circ} \mathrm{C}$ [95] and subsequently, a temperaturedependent model [96]. The best model showed a root mean square error of $0.148 \mathrm{log}$ units for the test set of 79 compounds and 133 data points.

Although ANNs have surprisingly vast number of applications, it appears that ANNs may also yield the best prediction results in development of QSAR and QSPR models when used in combination with other statistical methods. For example GA may be implemented in the optimization process during the network training in search for optimal data training set, such as demonstrated by a recent study on indole substitutions that could inhibit HIV-1 attachment found GA-ANNs the best model in comparison with MLR and MLR-ANN [71, 97]. As there are numerous potential combinations, further studies are needed on development of these statistical models based on the field of study and the nature of the problem in consideration.

\subsection{ANNs Application in Proteomics and Genomics}

Due to their ability to recognize patterns, ANNs are suitable for recognition of domains, classification of proteins, prediction of enzyme class, sequence classification of DNA/RNA and protein. These properties are especially useful in the study of the protein structure and function and could assist in prediction of protein tertiary structure such as a feed forward ANN consisting of six input and six hidden units with sigmoid transfer function developedby Murvai et. al. for the recognition of domains in protein sequence [98]. Cai and colleagues used ANNs for predicting secondary protein structure based on pair-coupled amino acid sequence and demonstrated that ANNs is a suitable method for prediction of the contents of prominent secondary structures of the proteins [99, 100]. Further, as ANNsare able to recognize the patterns based on the original sequences, it becomes possible to group large number of proteins by predefined classifications. A hierarchical network named PRED-CLASS have been used to classify proteins into four classes such as transmembrane, fibrous, globular and mixed proteins [101]. The PRED-CLASS trained using 50 protein sequences, correctly predicted 371 out of a set of 387 proteins with an accuracy of 96 percent. Artificial neural networks have been applied for predicting the separation of peptides in strong anion exchange chromatography. Such capability allows researchers to predict peptide separation 
and assist with required data mining steps such as protein identification [102].

Additionally, ANNs enable simulation of drug molecules and protein structures and help determine the folding and secondary structure of RNA strand [103, 104]. Also, ANN based algorithms have been used to identify, characterize and predict stabilization center elements from primary structure of single proteins and amino acid sequences of homologous proteins [105]. The stabilization center elements present in proteins stabilize protein structures by preventing their decay. The prediction of long chain fatty acid transport protein FadL topology [106], prediction of secondary structured of clostridialneuroprotein-C fragment [107], DNA/RNA and protein sequences analysis [108] are other applications where ANN technology has been exploited. The neural networks have been employed to predict eukaryotic protein phosphorylation sites [109], to recognize active sites and to predict enzyme class with high accuracy for novel protein structures [110], in peptide design and optimization [111], protein localization and sorting prediction $[112,113]$.

\subsection{ANNs Application in Pharmacokinetics}

It has been established that ANNs have wide applications in pharmacology, including in pharmacokinetics (PK) and pharmacodynamics (PD) - in monitoring multiple PK interactions in biological systems and in prediction of PD parameters such as clearance rates, protein bound fractions, and volume distribution $[114,115]$. Due to their computation versatility, ANNs have been utilized as analytical tool for population pharmacokinetic data analysis and was found superior to NONMEM with lesser average absolute errors and significantly lesser average prediction errors than NONMEM $[66,116]$. ANNs have been tested in prediction of Hussain et al. tested application of ANNs for prediction of PK parameters from those determined in animal studies [117]. Further, Ritchel et al. was able to predict human PK parameters using data set of physiochemical properties (protein binding, partition coefficients, dissociation constant, total clearance and volume of distribution) in combination with animal PK parameters [118]. Corrigan et al. applied neural network to predict gentamicin concentration in a general hospital population [119]. Their results indicated that neural networks offered some advantages over traditional dose prediction methods for gentamicin. Kenji et al. applied an ANN simulation to predict the pharmacokinetic of amino glycoside antibiotic using physiological measurement in patients with severe illness [120]. Recently, Moon et al. reported the PD model for dose determination of $\mathrm{HMG}-\mathrm{Co}-$ A-reductase inhibitors using ANNs [121]. An ANN model for dosing HMG-CoA-reductase inhibitors demonstrated an ability to predict appropriate dosing, but a larger sample size may be necessary for the development of a more accurate model. ANN analysis using standardized data showed reasonable predictive performance.

\subsection{Prediction Permeability of Skin and Blood Brain Barrier}

Development of an accurate skin permeability model is becoming increasingly important as skin has been more utilized in recent development of drug delivery methods. Also, the studies on absorption of toxic compounds through the skin are scarce with animal skin such as rodent and pig as a weak substitute [122]. Similar studies by AgatonovicKustrin et al. and Cheng et al. have published on use of ANNs in determining the quantitative structure-permeability relationship of penetration across skin or polydimethylsiloxane membranes as the model of skin permeation [123126]. A set of over 200 compounds and their maximum steady state flux was collected from the literature [127, 128]. In Agatonovic-Kustrin et al. study, twelve of 42 molecular descriptors were selected for ANN modeling of maximum steady-state flux by the use of genetic algorithm, that include molecular shape and size, inter-molecular interactions, hydrogen-bonding capacity of drugs, and conformational stability [123]. For the 12-descriptor neural network model, the training set relative means square error was 0.36 and the testing set relative mean square error was 0.59 . When the prediction power was evaluated using an external prediction set, the relative mean square error was 0.60 , indicating that the quality of the model would be ensured. Chen et al. study also confirmed the suitability of ANNs for prediction of skin permeability based on molecule descriptors such as molecular weight and partial charge [123].

Another method for predicting the human skin permeability $(\log \mathrm{K}$ p) of compounds has been developed based on three-dimensional molecular structure using a combination of molecular orbital (MO) calculations and ANN. For 92 compounds that was listed in the Flynn's data [129], their molecular descriptors, such as dipole moment, polarizability, sum of charges of nitrogen and oxygen atoms $(\operatorname{sum}(\mathrm{N}, \mathrm{O}))$, and sum of charges of hydrogen atoms bonding to nitrogen or oxygen atoms (sum $(\mathrm{H}))$, were calculated from MO calculations. The correlation between these molecular descriptors and log Kpwas examined using a feed-forward back-propagation neural network. To improve the generalization capability of a $\mathrm{ANN}$, the network was trained with input patterns given $5 \%$ random noise [130]. The neural network model with a configuration of 4-4-1 for input, hidden, and output layers was much superior to the conventional multiple linear regression model in terms of RMS errors (0.528 vs. 0.930). Moreover, a 'leave-one-out' cross-validation revealed that the neural network model could predict skin permeability with a reasonable accuracy (predictive relative mean square error of 0.669). The ANN modeling of skin permeability for 45 compounds based on MO-calculated descriptors has been performed by $\mathrm{Fu}$ et al, with external validation was conducted for eight compounds, the ANN model gave a mean prediction error of $2.6 \%$, whereas the prediction error of the multiple linear regression model with the same descriptors was 32.09\% [131].

Degim et al. [132] analyzed skin permeability of 40 compounds by an ANN and compared its predictability with the multiple linear regression model obtained by Pugh et al. [114]. According to the linear model of Pugh et al., the partial charges of the penetrants, their molecular weight, and their calculated octanol-water partition coefficient $\log \mathrm{P}_{\mathrm{oct} / \mathrm{w}}$ were used as molecular descriptors. While the linear equation gave a regression coefficient $\left(\mathrm{r}^{2}\right)$ of 0.672 , the ANN produced $\log \mathrm{K}_{\mathrm{p}}$ values that correlated well with the experimental ones $\left(\mathrm{r}^{2}=0.997\right)$. In addition, they experimentally determined human skin permeability for some compounds that have not been previously investigated, 
and found that their experimental data can be predicted well from the ANN model developed.

The ability of ANNs to recognize and predict patterns may be applied in studying the blood-brain distribution (BBD) of a compound, which is commonly expressed as logarithmic function of the ratio of brain concentration to blood concentration of a compound. Experimental values of each concentration, however, are time consuming and expensive to obtain [133]. Therefore, reliable prediction model is necessary, which is possible from the molecular structure of a compound and by QSAR/QSPR models.

Numerous models have been developed and published for prediction of BBD. Narayan and Gunturi have developed MLR method based model for BBD prediction by four descriptors of 88 compounds $(\mathrm{r}=0.8638, \mathrm{q}=0.8479$ and $\mathrm{SE}=0.3929$ ) [134]. In 2006, improved ANNs have been built based on chemical descriptors of the moleculesKatritzkyet.al. usedfive descriptors $\left(\mathrm{r}^{2}=0.781\right.$ and $\left.\mathrm{s}=0.123\right)$ and Garg and Verma used seven descriptors (for training $\mathrm{r}=0.90$ and for testing $\mathrm{r}=0.89)[135,136]$. A benchmark of predictive accuracy in the area of QSAR models for bloodbrain barrier (BBB) permeation prediction was established by Konovalov et al. in developing a predictive log BBQSAR model based on MLR method [137]. Afterward, Yan et al. carried out comparison study involving over 300 compounds by 19 global molecular descriptors, eight shape descriptors, 88 2D autocorrelation vectors, and 1024 3D property weighted RDF descriptors using MLR, SVM, and ANN [138]. ANN was found to have similar prediction abilities as MLR and SVM.

\subsection{Diagnosis of Disease}

ANNs have been applied in the diagnosis based on clinical chemical data of many diseases - cancers [139-141]; early diagnosis of lupus erythmatosus [142]; diagnosis of acute myocardial infarction [143, 144]; prediction of cardiovascular risk [145]; prediction of the development of pregnancy-induced hypertensive disorders [146]; diagnosis of Alzheimer's disease [147]; diagnosis of benign focal liver disease [148]; prediction of metabolic syndrome [149]; AIDS research and diagnosis [150]; Parkinsonian tremor [151]; urologic oncology [152]; diagnosis of pigmented skin lesions [153]; lung nodule detection [154]; prediction of outcome in epilepsy surgery [155]; and in assisting in making diagnosis decisions in emergency room [156].

In addition, ANNs have also been used to predict responses to pharmacotherapy. Cases in point, ANNs have been used to predict the response to hormonal treatment in metastatic breast cancer [157]. They can be used to predict the clinical outcome for individual patients. In contrast to conventional methods, the level of confidence for the predictions can reach $90 \%$ and more using ANNs. Such predictive power will be a big step towards individualized therapy in the future.

\section{CONCLUSIONS AND FUTURE PERSPECTIVES}

The application of ANN in medical decision making has been immensely successful especially as it applies to disease diagnosis, classification and modeling. The ANNs are newly developed strategies as an alternative to conventional modeling techniques. Applications of ANNs in the pharmaceutical field have been of increased interest due to their ability to model process that cannot be adequately represented using classical statistical methods. The ANNs do not need special computer as neural nets are described using mathematical models and implemented using ordinary computer software. Training time for networks is long but considerably advantageous. ANNs are an improvement over response surface methodology because they allow incorporation of literature and experimental data to solve common problems in pharmaceutical industry. It is capable of solving problems involving complex pattern recognition, which is advantageous in pharmaceutical product development. The use of artificial neural network in pharmaceutical research drug discovery is growing at a fast rate with very promising prospects.

Due to their capacity to learn, recognize patterns, and generalize, ANNs are a great tool in data analysis and modeling. The scope of current uses that ranges from spacial perceptions to chemical properties, activity, diagnosis and toxicology indicates the true potential of ANNs in analyzing the data and making predictions. This exciting versatility allows ANNs be utilized almost in any area of science requiring analysis of large, variable, and/or multivariate data; thus, applications of ANNs are expected to continue expanding into many more disciplines.

\begin{tabular}{|c|c|c|}
\hline \multicolumn{3}{|c|}{ ABBREVIATIONS } \\
\hline ADME & $=$ & $\begin{array}{l}\text { absorption, distribution, metabolism, and } \\
\text { excretion }\end{array}$ \\
\hline ANNs & $=$ & Artificial neural networks \\
\hline API & $=$ & active pharmaceutical ingredient \\
\hline GA & $=$ & generic algorithms \\
\hline HPLC & $=$ & high performance liquid chromatography \\
\hline MLR & $=$ & multiple linear regression \\
\hline PE & $=$ & processing units \\
\hline PD & $=$ & pharmacodynamics \\
\hline $\mathrm{PK}$ & $=$ & pharmacokinetics \\
\hline RDF & $=$ & radial distribution function \\
\hline RMS & $=$ & root mean squared error \\
\hline RSM & $=$ & response surface methodology \\
\hline QSAR & $=$ & $\begin{array}{l}\text { Quantitative } \\
\text { Relationships }\end{array}$ \\
\hline QSPR & $=$ & $\begin{array}{l}\text { Quantitative } \\
\text { Relationship }\end{array}$ \\
\hline SRR & $=$ & $=$ Structure Retention Relationship \\
\hline SVM & $=$ & support vector machine \\
\hline
\end{tabular}

\section{CONFLICT OF INTEREST}

The authors confirm that this article content has no conflicts of interest. 


\section{ACKNOWLEDGEMENTS}

Declared none.

\section{REFERENCES}

[1] J. Fu, Y. K. Jan, and M. Jones, "Development of intelligent model to determine favorable wheelchair tilt and recline angles for people with spinal cord injury," Conference proceedings : ... Annual International Conference of the IEEE Engineering in Medicine and Biology Society. IEEE Engineering in Medicine and Biology Society. Conference., vol. 2011, pp. 2045-8, 2011.

[2] J. Yang, H. Singh, E. L. Hines, F. Schlaghecken, D. D. Iliescu, M. S. Leeson, and N. G. Stocks, "Channel selection and classification of electroencephalogram signals: an artificial neural network and genetic algorithm-based approach," Artif. Intell. Med., vol. 55, pp. 117-26, Jun 2012.

[3] F. Bettella, D. Rasinski, and E. W. Knapp, "Protein secondary structure prediction with SPARROW," J. Chem. Inform. Model., vol. 52, pp. 545-56, Feb. 2012.

[4] B. Liu and Y. Jiang, "A multitarget training method for artificial neural network with application to computer-aided diagnosis," Med. Phys., vol. 40, p. 011908, Jan 2013.

[5] W. Zhao and C. E. Davis, "A modified artificial immune system based pattern recognition approach--an application to clinical diagnostics," Artif. Intell. Med., vol. 52, pp. 1-9, May 2011.

[6] M. Wesolowski and B. Suchacz, "Artificial neural networks: theoretical background and pharmaceutical applications: a review," J. AOAC Inter., vol. 95, pp. 652-68, May-Jun 2012.

[7] N. Minovski, S. Zuperl, V. Drgan, and M. Novic, "Assessment of applicability domain for multivariate counter-propagation artificial neural network predictive models by minimum Euclidean distance space analysis: A case study," Anal. Chim. Acta., vol. 759, pp. 2842, Jan 2013.

[8] R. Bartzatt, "Anti-inflammatory drugs and prediction of new structures by comparative analysis," Anti-inflamm. Anti-allergy Agents. Med. Chem., vol. 11, pp. 151-60, Sep 2012.

[9] J. S. Almeida, "Predictive non-linear modeling of complex data by artificial neural networks," Curr. Opin. Biotechnol., vol. 13, pp. 726, Feb 2002.

[10] S. Agatonovic-Kustrin and R. Beresford, "Basic concepts of artificial neural network (ANN) modeling and its application in pharmaceutical research," J. Pharm. Biomed. Anal., vol. 22, pp. 717-27, Jun 2000.

[11] D. R. JL. McClelland, Explorations in Parallel Distributed Processing. Cambridge: MIT Press, 1998.

[12] J. Bourquin, H. Schmidli, P. van Hoogevest, and H. Leuenberger, "Basic concepts of artificial neural networks (ANN) modeling in the application to pharmaceutical development," Pharm. Dev. Technol., vol. 2, pp. 95-109, May 1997.

[13] S. Ferrari and R. F. Stengel, "Smooth function approximation using neural networks," IEEE Trans Neural Netw (a publication of the IEEE Neural Networks Council), vol. 16, pp. 24-38, Jan 2005.

[14] M. N. Jadid and D. R. Fairbairn, "Neural-network applications in predicting moment-curvature parameters from experimental data," Eng. Appl. Artif. Intell., vol. 9, pp. 309-319, 1996.

[15] R. J. Erb, "Introduction to backpropagation neural network computation," Pharm. Res., vol. 10, pp. 165-70, Feb 1993.

[16] S. L. Goh and D. P. Mandic, "An augmented extended Kalman filter algorithm for complex-valued recurrent neural networks," Neural. Comput., vol. 19, pp. 1039-55, Apr 2007.

[17] F. Liu and M. J. Er, "A novel efficient learning algorithm for selfgenerating fuzzy neural network with applications," Int. J Neural. Syst., vol. 22, pp. 21-35, Feb 2012.

[18] S. Kirkpatrick, C. D. Gelatt, Jr., and M. P. Vecchi, "Optimization by simulated annealing," Science, vol. 220, pp. 671-80, May 13 1983.

[19] F. Shiratani and K. Yamamoto, "Deterministic annealing techniques for a discrete-time neural-network updating in a blocksequential mode," IEEE Trans. Neural Netw (a publication of the IEEE Neural. Netw Council)., vol. 9, pp. 345-53, 1998.

[20] K. Bouleimen and H. Lecocq, "A new efficient simulated annealing algorithm for the resource-constrained project scheduling problem and its multiple mode version," Eur. J. Oper. Res., vol. 149, pp. 268-281, 2003.
[21] H. M. Carpenter WC, "Understanding neural network approximations and polynomial approximations helps neural network performance," AI Expert. March., vol. 2, pp. 31-33, 1995.

[22] T. Aoyama, Y. Suzuki, and H. Ichikawa, "Neural networks applied to quantitative structure-activity relationship analysis," J. Medic. Chem., vol. 33, pp. 2583-90, Sep 1990.

[23] C. W. Lim, S. Fujiwara, F. Yamashita, and M. Hashida, "Prediction of human skin permeability using a combination of molecular orbital calculations and artificial neural network," Biol. Pharm. Bull., vol. 25, pp. 361-6, Mar 2002.

[24] S. Fujiwara, F. Yamashita, and M. Hashida, "Prediction of Caco-2 cell permeability using a combination of MO-calculation and neural network," Int. J. Pharm., vol. 237, pp. 95-105, Apr 2002.

[25] K. Takayama, A. Morva, M. Fujikawa, Y. Hattori, Y. Obata, and T. Nagai, "Formula optimization of theophylline controlled-release tablet based on artificial neural networks," J. Control. Release. Soc., vol. 68, pp. 175-86, Aug 2000.

[26] J. Wu, J. Mei, S. Wen, S. Liao, J. Chen, and Y. Shen, "A selfadaptive genetic algorithm-artificial neural network algorithm with leave-one-out cross validation for descriptor selection in QSAR study," J. Comput. Chem., vol. 31, pp. 1956-68, Jul 2010.

[27] A. Pukrittayakamee, M. Hagan, L. Raff, S. T. S. Bukkapatnam, and R. Komanduri, "Practical training framework for fitting a function and its derivatives," Neural. Netw. IEEE. Trans., vol. 22, pp. 936$947,2011$.

[28] C. C. Chen and D. Jasnow, "Mean-field theory of a plastic network of integrate-and-fire neurons," Phys. Rev. E Stat. Nonlin. Soft. Matter. Phys., vol. 81, p. 011907, Jan 2010.

[29] N. R. Luque, J. A. Garrido, J. Ralli, J. J. Laredo, and E. Ros, "From sensors to spikes: evolving receptive fields to enhance sensorimotor information in a robot-arm," Int. J. Neural. Syst., vol. 22, p. 1250013, Aug 2012.

[30] B. Inden, Y. Jin, R. Haschke, and H. Ritter, "Evolving neural fields for problems with large input and output spaces," Neural. Netw. vol. 28, pp. 24-39, Apr 2012.

[31] Y. Ni, W. Xiao, and S. Kokot, "Application of chemometrics methods for the simultaneous kinetic spectrophotometric determination of aminocarb and carbaryl in vegetable and water samples," J. Hazard. Mater., vol. 168, pp. 1239-45, Sep 2009.

[32] M. Hasani, M. Moloudi, and F. Emami, "Spectrophotometric resolution of ternary mixtures of tryptophan, tyrosine, and histidine with the aid of principal component-artificial neural network models," Anal. Biochem., vol. 370, pp. 68-76, Nov 2007.

[33] L. Bai, H. Zhang, H. Wang, J. Li, and L. Lu, "Analysis of ultraviolet absorption spectrum of Chinese herbal medicine-Cortex Fraxini by double ANN," Spectrochim. Acta A., vol. 65, pp. 863-8, Nov 2006.

[34] L. Zhu, S. H. Shabbir, and E. V. Anslyn, "Two methods for the determination of enantiomeric excess and concentration of a chiral sample with a single spectroscopic measurement," Chemistry., vol. 13, pp. 99-104, 2007.

[35] S. Agatonovic-Kustrin, I. G. Tucker, and D. Schmierer, "Solid state assay of ranitidine $\mathrm{HCl}$ as a bulk drug and as active ingredient in tablets using DRIFT spectroscopy with artificial neural networks," Pharm. Res., vol. 16, pp. 1477-82, Sep 1999.

[36] S. Agatonovic-Kustrin, V. Wu, T. Rades, D. Saville, and I. G. Tucker, "Powder diffractometric assay of two polymorphic forms of ranitidine hydrochloride," Int. J. Pharm., vol. 184, pp. 107-14, Jul 1999.

[37] S. Agatonovic-Kustrin, V. Wu, T. Rades, D. Saville, and I. G. Tucker, "Ranitidine hydrochloride X-ray assay using a neural network," J. Pharm. Biomed. Anal., vol. 22, pp. 985-92, Jul 2000.

[38] T. Baczek, A. Bucinski, A. R. Ivanov, and R. Kaliszan, "Artificial neural network analysis for evaluation of peptide MS/MS spectra in proteomics," Anal. Chem., vol. 76, pp. 1726-32, Mar 2004.

[39] J. E. Madden, N. Avdalovic, P. R. Haddad, and J. Havel, "Prediction of retention times for anions in linear gradient elution ion chromatography with hydroxide eluents using artificial neural networks," J. Chromatogr. A., vol. 910, pp. 173-9, Feb 2001.

[40] S. C. Stefanovic, T. Bolanca, M. Lusa, S. Ukic, and M. Rogosic, "Multi-criteria decision making development of ion chromatographic method for determination of inorganic anions in oilfield waters based on artificial neural networks retention model," Anal. Chim. Acta., vol. 716, pp. 145-54, Feb 2012.

[41] T. Bolanca, S. Cerjan-Stefanovic, M. Lusa, S. Ukic, and M. Rogosic, "Evaluation of separation in gradient elution ion 
chromatography by combining several retention models and objective functions," J. Separ. Sci., vol. 31, pp. 705-13, Mar 2008.

[42] S. Y. Tham and S. Agatonovic-Kustrin, "Application of the artificial neural network in quantitative structure-gradient elution retention relationship of phenylthiocarbamyl amino acids derivatives," J. Pharm. Biomed. Anal., vol. 28, pp. 581-90, May 2002.

[43] S. Agatonovic-Kustrin, M. Zecevic, L. Zivanovic, and I. G. Tucker, "Application of artificial neural networks in HPLC method development," J. Pharm. Biomed. Anal., vol. 17, pp. 69-76, May 1998.

[44] J. A. Lewis, D. C. Lommen, W. D. Raddatz, J. W. Dolan, L. R. Snyder, and I. Molnar, "Computer simulation for the prediction of separation as a function of $\mathrm{pH}$ for reversed-phase high-performance liquid chromatography. I. Accuracy of a theory-based model," $J$. Chromatogr., vol. 592, pp. 183-95, Feb 1992.

[45] J. A. Lewis, J. W. Dolan, L. R. Snyder, and I. Molnar, "Computer simulation for the prediction of separation as a function of $\mathrm{pH}$ for reversed-phase high-performance liquid chromatography. II. Resolution as a function of simultaneous change in $\mathrm{pH}$ and solvent strength," J. Chromatogr., vol. 592, pp. 197-208, Feb 1992.

[46] M. T. Hearn, M. I. Aguilar, C. T. Mant, and R. S. Hodges, "Highperformance liquid chromatography of amino acids, peptides and proteins. LXXXV. Evaluation of the use of hydrophobicity coefficients for the prediction of peptide elution profiles," $J$. Chromatogr., vol. 438, pp. 197-210, Apr 1988.

[47] S. Agatonovic-Kustrin, M. Zecevic, and L. Zivanovic, "Use of ANN modelling in structure--retention relationships of diuretics in RP-HPLC," J. Pharm. Biomed. Anal., vol. 21, pp. 95-103, Oct 1999.

[48] G. Chen, J. Li, S. Zhang, C. Song, G. Li, Z. Sun, Y. Suo, and J. You, "A sensitive and efficient method to systematically detect two biophenols in medicinal herb, herbal products and rat plasma based on thorough study of derivatization and its convenient application to pharmacokinetics with semi-automated device," J. Chromatogr. A., vol. 1249, pp. 190-200, Aug 32012.

[49] N. K. Ebube, G. Owusu-Ababio, and C. M. Adeyeye, "Preformulation studies and characterization of the physicochemical properties of amorphous polymers using artificial neural networks," Int. J. Pharm., vol. 196, pp. 27-35, Feb 2000.

[50] Y. Onuki, S. Kawai, H. Arai, J. Maeda, K. Takagaki, and K. Takayama, "Contribution of the physicochemical properties of active pharmaceutical ingredients to tablet properties identified by ensemble artificial neural networks and kohonen's self-organizing maps," J. Pharm. Sci., vol. 101, pp. 2372-2381, 2012.

[51] B. D. Glass, S. Agatonovic-Kustrin, and M. H. Wisch, "Artificial neural networks to optimize formulation components of a fixeddose combination of rifampicin, isoniazid and pyrazinamide in a microemulsion," Curr. Drug. Discov. Technol., vol. 2, pp. 195-201, Sep 2005.

[52] J. Takahara, "Multi-objective simultaneous optimization technique based on an artificial neural network in sustained release formulations," J. Controll. Release, vol. 49, p. 11, 1997.

[53] J. Takahara, K. Takayama, K. Isowa, and T. Nagai, "Multiobjective simultaneous optimization based on artificial neural network in a ketoprofen hydrogel formula containing Oethylmenthol as a percutaneous absorption enhancer," Int. J. Pharm., vol. 158, pp. 203-210, 1997.

[54] A. S. Hussain, X. Q. Yu, and R. D. Johnson, "Application of neural computing in pharmaceutical product development," Pharm. Res., vol. 8, pp. 1248-52, Oct 1991 .

[55] S. Ibric, M. Jovanovic, Z. Djuric, J. Parojcic, and L. Solomun, "The application of generalized regression neural network in the modeling and optimization of aspirin extended release tablets with Eudragit RS PO as matrix substance," J. Controll. Release. vol. 82, pp. 213-222, Aug 2002.

[56] S. Ibric, M. Jovanovic, Z. Djuric, J. Parojcic, S. D. Petrovic, L. Solomun, and B. Stupar, "Artificial neural networks in the modeling and optimization of aspirin extended release tablets with Eudragit L 100 as matrix substance," AAPS. Pharm. Sci. Tech. , vol. 4, p. E9, 2003.

[57] D. Zupançiç Boziç, F. Vreçer, and F. Kozjek, "Optimization of diclofenac sodium dissolution from sustained release formulations using an artificial neural network," Eur. J. Pharm. Sci., vol. 5, pp. 163-169, 1997.
[58] T. Wu, W. Pan, J. Chen, and R. Zhang, "Formulation optimization technique based on artificial neural network in salbutamol sulfate osmotic pump tablets," Drug. Dev. Industr. Pharm., vol. 26, pp. 211-5, Feb 2000.

[59] K. Takayama, M. Fujikawa, and T. Nagai, "Artificial neural network as a novel method to optimize pharmaceutical formulations," Pharm. Res., vol. 16, pp. 1-6, Jan 1999.

[60] P. Barmpalexis, K. Kachrimanis, and E. Georgarakis, "Solid dispersions in the development of a nimodipine floating tablet formulation and optimization by artificial neural networks and genetic programming," Eur. J. Pharm. Biopharm., vol. 77, pp. 122 $131,2011$.

[61] Y. Chen, T. W. McCall, A. R. Baichwal, and M. C. Meyer, "The application of an artificial neural network and pharmacokinetic simulations in the design of controlled-release dosage forms," $J$ Controll. Release., vol. 59, pp. 33-41, May1999.

[62] S. Vaithiyalingam and M. A. Khan, "Optimization and characterization of controlled release multi-particulate beads formulated with a customized cellulose acetate butyrate dispersion," Int. J. Pharm., vol. 234, pp. 179-93, Mar 2002.

[63] E. Aktas, H. Eroglu, U. Kockan, and L. Oner, "Systematic development of $\mathrm{pH}$-independent controlled release tablets of carvedilol using central composite design and artificial neural networks," Drug. Dev. Industr. Pharmacy., Jul 2012.

[64] A. Patel, T. Mehta, M. Patel, K. Patel, and N. Patel, "Design Porosity Osmotic Tablet for Delivering Low and $\mathrm{pH}$-Dependent Soluble Drug Using An Artificial Neural Network," Curr. Drug. Deliv., vol. 9, pp. 459-67, Sep 2012.

[65] J. A. Dowell, A. Hussain, J. Devane, and D. Young, "Artificial neural networks applied to the in vitro-in vivo correlation of an extended-release formulation: initial trials and experience," $J$. Pharm. Sci., vol. 88, pp. 154-60, Jan 1999.

[66] J. V. Gobburu and E. P. Chen, "Artificial neural networks as a novel approach to integrated pharmacokinetic-pharmacodynamic analysis," J. Pharm. Sci., vol. 85, pp. 505-10, May 1996.

[67] C. H. Richardson, M. de Matas, H. Hosker, R. Mukherjee, I. Wong, and H. Chrystyn, "Determination of the relative bioavailability of salbutamol to the lungs following inhalation from dry powder inhaler formulations containing drug substance manufactured by supercritical fluids and micronization," Pharm. Res., vol. 24, pp. 2008-17, Nov 2007.

[68] M. de Matas, Q. Shao, C. H. Richardson, and H. Chrystyn, "Evaluation of in vitro in vivo correlations for dry powder inhaler delivery using artificial neural networks," Eur. J. Pharm. Sci., vol. 33, pp. 80-90, 2008.

[69] P. Paixão, L. s. F. Gouveia, and J. A. G. Morais, "Prediction of the in vitro intrinsic clearance determined in suspensions of human hepatocytes by using artificial neural networks," Eur. J. Pharm. Sci., vol. 39, pp. 310-321, 2010.

[70] K. Z. Myint, L. Wang, Q. Tong, and X. Q. Xie, "Molecular fingerprint-based artificial neural networks QSAR for ligand biological activity predictions," Mol. Pharm., vol. 9, pp. 2912-23, Oct 2012.

[71] M. Nirouei, G. Ghasemi, P. Abdolmaleki, A. Tavakoli, and S. Shariati, "Linear and non-linear quantitative structure-activity relationship models on indole substitution patterns as inhibitors of HIV-1 attachment," Indian J. Biochem. Biophys., vol. 49, pp. 20210, Jun 2012.

[72] Y. Uesawa, K. Mohri, M. Kawase, M. Ishihara, and H. Sakagami, "Quantitative structure-activity relationship (QSAR) analysis of tumor-specificity of 1,2,3,4-tetrahydroisoquinoline derivatives," Anticancer Res., vol. 31, pp. 4231-8, Dec 2011.

[73] J. C. Dearden and M. Hewitt, "QSAR modelling of bioconcentration factor using hydrophobicity, hydrogen bonding and topological descriptors," SAR. QSAR Environ. Res., vol. 21, pp. 671-80, Oct 2010 .

[74] J. V. Gobburu and W. H. Shelver, "Quantitative structurepharmacokinetic relationships (QSPR) of beta blockers derived using neural networks," J. Pharm. Sci., vol. 84, pp. 862-5, Jul 1995.

[75] I. S. Nestorov, S. T. Hadjitodorov, I. Petrov, and M. Rowland, "Empirical versus mechanistic modelling: comparison of an artificial neural network to a mechanistically based model for quantitative structure pharmacokinetic relationships of a homologous series of barbiturates," AAPS. Pharm. Sci., vol. 1, p. E17, 1999. 
[76] J. Jaen-Oltra, M. T. Salabert-Salvador, F. J. Garcia-March, F. Perez-Gimenez, and F. Tomas-Vert, "Artificial neural network applied to prediction of fluorquinolone antibacterial activity by topological methods," J. Med. Chem., vol. 43, pp. 1143-8, Mar 2000.

[77] M. Torrent, D. Andreu, V. M. Nogues, and E. Boix, "Connecting peptide physicochemical and antimicrobial properties by a rational prediction model," PloS One. , vol. 6, p. e16968, 2011.

[78] M. D. Ertürk, M. T. r. Sa $\sqrt{ }$ an, M. Novic, and N. Minovski, "Quantitative structure, Äiactivity relationships (QSARs) using the novel marine algal toxicity data of phenols," J. Mol. Graph. Model., vol. 38, pp. 90-100, 2012.

[79] R. Sabet, A. Fassihi, B. Hemmateenejad, L. Saghaei, R. Miri, and M. Gholami, "Computer-aided design of novel antibacterial 3hydroxypyridine-4-ones: application of QSAR methods based on the MOLMAP approach," J. Comput. Aid. Mol. Des., vol. 26, pp. 349-61, Mar 2012.

[80] M. Koba, "Application of artificial neural networks for the prediction of antitumor activity of a series of acridinone derivatives," Med. Chem., vol. 8, pp. 309-19, May 2012.

[81] B. B. Andreas Breindl, Timothy Clark, Robert C. Glen, "Prediction of the n-Octanol/water partition coefficient, logp, using a combination of semiempirical mo-calculations and a neural network," J. Mol. Model., vol. 3, pp. 142-155, 1997.

[82] D. Eros, I. Kovesdi, L. Orfi, K. Takacs-Novak, G. Acsady, and G. Keri, "Reliability of $\log \mathrm{P}$ predictions based on calculated molecular descriptors: a critical review," Curr. Med. Chem., vol. 9, pp. 181929 , Oct 2002.

[83] A. J. Wilczynska-Piliszek, S. Piliszek, and J. Falandysz, "Use of quantitative-structure property relationship (QSPR) and artificial neural network (ANN) based approaches for estimating the octanol-water partition coefficients of the 209 chlorinated transazobenzene congeners," J. Environ. Sci. Health. B., vol. 47, pp. 111-28, 2012.

[84] H. Noorizadeh, A. Farmany, M. Noorizadeh, and M. Kohzadi, "Prediction of polar surface area of drug molecules: A QSPR approach," Drug. Test. Anal., May 2011.

[85] B. Louis, V. K. Agrawal, and P. V. Khadikar, "Prediction of intrinsic solubility of generic drugs using MLR, ANN and SVM analyses," Eur. J. Med. Chem., vol. 45, pp. 4018-25, Sep 2010.

[86] D. Cherqaoui and D. Villemin, "Use of a neural network to determine the boiling point of alkanes," J. Chem. Soc., Faraday. Trans., vol. 90, pp. 97-102, 1994.

[87] A. Yan, X. Chen, R. Zhang, M. Liu, Z. Hu, and B. T. Fan, "Predicting the standard enthalpy (deltaH0f) and entropy (S0) of alkanes by artificial neural networks," SAR. QSAR. Environ. Res., vol. 11 , pp. $235-44,2000$.

[88] S. Liu, "Neural Network-Topological Indices Approach to the Prediction of Properties of Alkene," J. Chem. Inform. Comput Sci., vol. 37, p. 1146, 1997.

[89] G. Espinosa, D. Yaffe, Y. Cohen, A. Arenas, and F. Giralt, "Neural network based quantitative structural property relations (QSPRs) for predicting boiling points of aliphatic hydrocarbons," J. Chem. Inform. Comput. Sci., vol. 40, pp. 859-79, May 2000.

[90] E. S. Goll and P. C. Jurs, "Prediction of Vapor Pressures of Hydrocarbons and Halohydrocarbons from Molecular Structure with a Computational Neural Network Model," J. Chem. Inform. Comput. Sci., vol. 39, pp. 1081-1089, 1999/11/01 1999.

[91] S. Piliszek, A. J. Wilczynska-Piliszek, and J. Falandysz, "Prediction of subcooled vapor pressures (log PL) of 399 polychlorinated trans-azoxybenzenes by using the QSPR and ANN approach," J. Environ. Sci. Health. A., vol. 47, pp. 450-61, 2012.

[92] D. Yaffe, Y. Cohen, G. Espinosa, A. Arenas, and F. Giralt, "Fuzzy ARTMAP and back-propagation neural networks based quantitative structure-property relationships (QSPRs) for octanolwater partition coefficient of organic compounds," J. Chem. Inform. Comput. Sci.,, vol. 42, pp. 162-83, Mar-Apr 2002.

[93] S. Sild and M. Karelson, "A General QSPR Treatment for Dielectric Constants of Organic Compounds," J Chem. Inform. Comput. Sci., vol. 42, pp. 360-367, 2002/03/01 2002.

[94] J. Tetteh, T. Suzuki, E. Metcalfe, and S. Howells, "Quantitative Structure,àiProperty Relationships for the Estimation of Boiling Point and Flash Point Using a Radial Basis Function Neural Network," J. Chem. Inform. Comput. Sci., vol. 39, pp. 491-507, 1999/05/01 1999.
[95] T. Suzuki, R.-U. Ebert, and G. Schrmann, "Development of Both Linear and Nonlinear Methods To Predict the Liquid Viscosity at $20{ }^{\circ} \mathrm{C}$ of Organic Compounds," J. Chem. Inform. Comput. Sci., vol. 37, pp. 1122-1128, 1997/11/01 1997.

[96] T. Suzuki, R. U. Ebert, and G. Schuurmann, "Application of neural networks to modeling and estimating temperature-dependent liquid viscosity of organic compounds," J. Chem. Inform. Comput. Sci., vol. 41, pp. 776-90, May-Jun 2001.

[97] F. H. F. Leung, H. K. Lam, S. H. Ling, and P. K. S. Tam, "Tuning of the structure and parameters of a neural network using an improved genetic algorithm," Neural. Netw., IEEE Trans., vol. 14, pp. 79-88, 2003

[98] J. Murvai, K. Vlahovicek, C. Szepesvari, and S. Pongor, "Prediction of protein functional domains from sequences using artificial neural networks," Genome. Res., vol. 11, pp. 1410-7, Aug 2001.

[99] Y.-D. Cai, X.-J. Liu, X.-b. Xu, and K.-C. Chou, "Artificial neural network method for predicting protein secondary structure content," Comput. Chem., vol. 26, pp. 347-350, 2002.

[100] Y. D. Cai, X. J. Liu, and K. C. Chou, "Prediction of protein secondary structure content by artificial neural network," $J$. Comput. Chem., vol. 24, pp. 727-31, Apr 2003.

[101] C. Pasquier, V. J. Promponas, and S. J. Hamodrakas, "PREDCLASS: cascading neural networks for generalized protein classification and genome-wide applications," Protein., vol. 44, pp. 361-9, Aug 2001.

[102] C. Oh, S. H. Zak, H. Mirzaei, C. Buck, F. E. Regnier, and X Zhang, "Neural network prediction of peptide separation in strong anion exchange chromatography," Bioinformatic., vol. 23, pp. 1148, Jan 12007.

[103] D. J. Livingstone, D. T. Manallack, and I. V. Tetko, "Data modelling with neural networks: Advantages and limitations," $J$. Comput. Aid. Mol. Des., vol. 11, pp. 135-142, 1997.

[104] C. Reidys, P. F. Stadler, and P. Schuster, "Generic properties of combinatory maps: neutral networks of RNA secondary structures," Bull. Math. Biol., vol. 59, pp. 339-97, Mar 1997.

[105] Z. Dosztanyi, A. Fiser, and I. Simon, "Stabilization centers in proteins: identification, characterization and predictions," J. Mol. Biol., vol. 272, pp. 597-612, Oct 1997.

[106] G. Cristalli, C. C. DiRusso, and P. N. Black, "The amino-terminal region of the long-chain fatty acid transport protein FadL contains an externally exposed domain required for bacteriophage T2 binding," Arch. Biochem. Biophys., vol. 377, pp. 324-33, May 2000 .

[107] F. J. Lebeda, T. C. Umland, M. Sax, and M. A. Olson, "Accuracy of secondary structure and solvent accessibility predictions for a clostridial neurotoxin C-fragment," J. Protein. Chem., vol. 17, pp. 311-8, May 1998.

[108] C. H. Wu, "Artificial neural networks for molecular sequence analysis," Comput. Chem., vol. 21, pp. 237-56, 1997.

[109] N. Blom, S. Gammeltoft, and S. Brunak, "Sequence and structurebased prediction of eukaryotic protein phosphorylation sites," $J$. Mol. Biol., vol. 294, pp. 1351-62, Dec 1999.

[110] M. Stahl, C. Taroni, and G. Schneider, "Mapping of protein surface cavities and prediction of enzyme class by a self-organizing neural network," Protein. Eng., vol. 13, pp. 83-8, Feb 2000.

[111] S. Bandholtz, J. Wichard, R. Kuhne, and C. Grotzinger, "Molecular evolution of a peptide GPCR ligand driven by artificial neural networks," PloS One., vol. 7, p. e36948, 2012.

[112] D. Mukherjee, C. B. Hanna, and R. C. Aguilar, "Artificial neural network for the prediction of tyrosine-based sorting signal recognition by adaptor complexes," J. Biomed. Biotechnol., vol. 2012, p. 498031, 2012.

[113] C. Mooney, Y. H. Wang, and G. Pollastri, "SCLpred: protein subcellular localization prediction by N-to-1 neural networks," Bioinformatic., vol. 27, pp. 2812-9, Oct 2011.

[114] W. J. Pugh, I. T. Degim, and J. Hadgraft, "Epidermal permeabilitypenetrant structure relationships: 4, QSAR of permeant diffusion across human stratum corneum in terms of molecular weight, Hbonding and electronic charge," Int. J. Pharm., vol. 197, pp. 20311, Mar 2000.

[115] S. H. Haidar, S. B. Johnson, M. J. Fossler, and A. S. Hussain, "Modeling the Pharmacokinetics and Pharmacodynamics of a Unique Oral Hypoglycemic Agent Using Neural Networks," Pharm. Res., vol. 19, pp. 87-91, 2002. 
[116] H. H. Chow, K. M. Tolle, D. J. Roe, V. Elsberry, and H. Chen, "Application of neural networks to population pharmacokinetic data analysis," J. Pharm. Sci., vol. 86, pp. 840-5, Jul 1997.

[117] A. S. Hussain, R. D. Johnson, N. N. Vachharajani, and W. A. Ritschel, "Feasibility of developing a neural network for prediction of human pharmacokinetic parameters from animal data," Pharm. Res., vol. 10, pp. 466-9, Mar 1993.

[118] W. A. Ritschel, R. Akileswaran, and A. S. Hussain, "Application of neural networks for the prediction of human pharmacokinetic parameters," Method. Find. Exp. Clin., vol. 17, pp. 629-43, Nov 1995.

[119] B. W. Corrigan, P. R. Mayo, and F. Jamali, "Application of a neural network for gentamicin concentration prediction in a general hospital population," Ther. Drug. Monit., vol. 19, pp. 25-8, Feb 1997.

[120] S. Yamamura, K. Nishizawa, M. Hirano, Y. Momose, and A. Kimura, "Prediction of plasma levels of aminoglycoside antibiotic in patients with severe illness by means of an artificial neural network simulator," J Pharmacy. Pharm. Sci., (a publication of the Canadian Society for Pharmaceutical Sciences, Societe canadienne des sciences pharmaceutiques), vol. 1, pp. 95-101, Sep-Dec 1998.

[121] A. Moon and T. Smith, "A preliminary evaluation of neural network analysis for pharmacodynamic modeling of the dosing of the hydroxymethylglutaryl coenzyme A-reductase inhibitors simvastatin and atorvastatin," Clin. Ther., vol. 24, pp. 653-61, Apr 2002.

[122] G. P. Moss, Y. Sun, S. C. Wilkinson, N. Davey, R. Adams, G. P. Martin, M. Prapopopolou, and M. B. Brown, "The application and limitations of mathematical modelling in the prediction of permeability across mammalian skin and polydimethylsiloxane membranes," J. Pharm. Pharmacol., vol. 63, pp. 1411-27, Nov 2011.

[123] S. Agatonovic-Kustrin, R. Beresford, and A. P. Yusof, "ANN modeling of the penetration across a polydimethylsiloxane membrane from theoretically derived molecular descriptors," $J$. Pharm. Biomed. Anal., vol. 26, pp. 241-54, Sep 2001.

[124] W. J. Addicks, G. L. Flynn, N. Weiner, and C. M. Chiang, "Drug transport from thin applications of topical dosage forms: development of methodology," Pharm. Res., vol. 5, pp. 377-82, Jun 1988.

[125] W. E. Jetzer, A. S. Huq, N. F. Ho, G. L. Flynn, N. Duraiswamy, and L. Condie, Jr., "Permeation of mouse skin and silicone rubber membranes by phenols: relationship to in vitro partitioning," $J$. Pharm. Sci., vol. 75, pp. 1098-103, Nov 1986.

[126] L. J. Chen, G. P. Lian, and L. J. Han, "Prediction of human skin permeability using artificial neural network (ANN) modeling," Acta Pharm. Sin., vol. 28, pp. 591-600, Apr 2007.

[127] Y. Chen, P. Vayumhasuwan, and L. E. Matheson, "Prediction of flux through polydimethylsiloxane membranes using atomic charge calculations: Application to an extended data set," Int. J. Pharm., vol. 137, pp. 149-158, 1996.

[128] M. T. Cronin, J. C. Dearden, R. Gupta, and G. P. Moss, "An investigation of the mechanism of flux across polydimethylsiloxane membranes by use of quantitative structure-permeability relationships," J. Pharm. Pharmacol. vol. 50, pp. 143-52, Feb 1998.

[129] G. L. Flynn, Ed., Physicochemical determinants of skin absorption (Principles of route to route extrapolation for risk assessment. New York: Elsevier Science, 1990.

[130] L. Holmstrom and P. Koistinen, "Using additive noise in backpropagation training," IEEE. Trans. Neural. Netw., vol. 3, pp. 2438, 1992.

[131] X. C. Fu, X. W. Ma, and W. Q. Liang, "Prediction of skin permeability using an artificial neural network," Die Pharm., vol. 57, pp. 655-6, Sep 2002.

[132] T. Degim, J. Hadgraft, S. Ilbasmis, and Y. Ozkan, "Prediction of skin penetration using artificial neural network (ANN) modeling," J. Pharm. Sci., vol. 92, pp. 656-64, Mar 2003.

[133] Z. Wang, A. Yan, and J. Li, "In Silico Prediction of Blood Brain Barrier Permeability: A Support Vector Machine Model," in Interdisciplinary Research and Applications in Bioinformatics, Computational Biology, and Environmental Sciences: IGI Global, 2011, pp. 155-171.

[134] Narayanan, "In silico ADME modelling: Prediction models for blood-brain barrier permeation using a systematic variable selection method," Bioorg. Med. Chem., vol. 13, p. 3017, 2005.
[135] Katritzky, "Correlation of blood-brain penetration using structural descriptors," Bioorg. Med. Chem., vol. 14, p. 4888, 2006.

[136] Garg, "In silico prediction of blood brain barrier permeability: An artificial neural network model," J. Chem. Inform. Model., vol. 46, p. $289,2006$.

[137] Konovalov, "Benchmarking of QSAR models for blood-brain barrier permeation," J. Chem. Inform. Model., vol. 47, p. 1648, 2007

[138] A. Yan, H. Liang, Y. Chong, X. Nie, and C. Yu, "In-silico prediction of blood-brain barrier permeability," SAR. QSAR Environ. Res., Oct 242012.

[139] Y. Shan, R. Zhao, G. Xu, H. M. Liebich, and Y. Zhang, "Application of probabilistic neural network in the clinical diagnosis of cancers based on clinical chemistry data," Anal. Chim Acta., vol. 471, pp. 77-86, 2002.

[140] J. Sachdeva, V. Kumar, I. Gupta, N. Khandelwal, and C. K. Ahuja, "A dual neural network ensemble approach for multiclass brain tumor classification," Int. J. Numer. Method. Biomed. Eng., vol. 28 pp. 1107-20, Nov 2012.

[141] A. Giordano, M. Giuliano, M. De Laurentiis, A. Eleuteri, F. Iorio, R. Tagliaferri, G. N. Hortobagyi, L. Pusztai, S. De Placido, K. Hess, M. Cristofanilli, and J. M. Reuben, "Artificial neural network analysis of circulating tumor cells in metastatic breast cancer patients," Breast. Cancer. Res. Treat., vol. 129, pp. 451-8, Sep 2011.

[142] M. H. Bahari, M. Mahmoudi, A. Azemi, M. M. Mirsalehi, and M. Khademi, "Early diagnosis of systemic lupus erythmatosus using ANN models of dsDNA binding antibody sequence data," Bioinformation., vol. 5, pp. 58-61, 2010.

[143] S. M. Pedersen, J. S. Jorgensen, and J. B. Pedersen, "Use of neural networks to diagnose acute myocardial infarction. II. A clinical application," Clin. Chem., vol. 42, pp. 613-7, Apr 1996.

[144] W. G. Baxt, "Analysis of the clinical variables driving decision in an artificial neural network trained to identify the presence of myocardial infarction," Annal. Emerg. Med., vol. 21, pp. 1439-44, Dec 1992.

[145] G. Leoncini, G. Sacchi, F. Viazzi, M. Ravera, D. Parodi, E. Ratto, S. Vettoretti, C. Tomolillo, G. Deferrari, and R. Pontremoli, "Microalbuminuria identifies overall cardiovascular risk in essential hypertension: an artificial neural network-based approach," J. Hypertens., vol. 20, pp. 1315-21, Jul 2002.

[146] G. Mello, E. Parretti, A. Ognibene, F. Mecacci, R. Cioni, G. Scarselli, and G. Messeri, "Prediction of the development of pregnancy-induced hypertensive disorders in high-risk pregnant women by artificial neural networks," Clinical chemistry and laboratory medicine : CCLM / FESCC, vol. 39, pp. 801-5, Sep 2001.

[147] X. Deng, K. Li, and S. Liu, "Preliminary study on application of artificial neural network to the diagnosis of Alzheimer's disease with magnetic resonance imaging," Chin. Med. J., vol. 112, pp. 232-7, Mar 1999.

[148] K. Rudzki, M. Hartleb, T. Sadowski, and J. Rudzka, "Focal liver disease: neural network-aided diagnosis based on clinical and laboratory data," Gastroenterol. Clin. Biol., vol. 21, pp. 98-102, 1997.

[149] H. Hirose, T. Takayama, S. Hozawa, T. Hibi, and I. Saito, "Prediction of metabolic syndrome using artificial neural network system based on clinical data including insulin resistance index and serum adiponectin," Comput. Biol. Med., vol. 41, pp. 1051-6, Nov 2011

[150] S. Sardari and D. Sardari, "Applications of artificial neural network in AIDS research and therapy," Curr. Pharm. Des., vol. 8, pp. 659$70,2002$.

[151] R. Edwards, A. Beuter, and L. Glass, "Parkinsonian tremor and simplification in network dynamics," Bull. Math. Biol., vol. 61, pp. 157-77, Jan 1999.

[152] T. H. Douglas and J. W. Moul, "Applications of neural networks in urologic oncology," Semin. Urol. Oncol., vol. 16, pp. 35-9, Feb 1998.

[153] P. Rubegni, G. Cevenini, M. Burroni, R. Perotti, G. Dell'Eva, P Sbano, C. Miracco, P. Luzi, P. Tosi, P. Barbini, and L. Andreassi, "Automated diagnosis of pigmented skin lesions," Int. J. Cancer., vol. 101, pp. 576-80, Oct 202002.

[154] M. G. Penedo, M. J. Carreira, A. Mosquera, and D. Cabello, "Computer-aided diagnosis: a neural-network-based approach to 
lung nodule detection," IEEE. Trans. Med. Imag., vol. 17, pp. 87280, Dec 1998.

[155] J. E. Arle, K. Perrine, O. Devinsky, and W. K. Doyle, "Neural network analysis of preoperative variables and outcome in epilepsy surgery," J. Neurosurg., vol. 90, pp. 998-1004, Jun 1999.

[156] L. Grigull and W. M. Lechner, "Supporting diagnostic decisions using hybrid and complementary data mining applications: a pilot study in the pediatric emergency department," Pediatr. Res., vol. 71, pp. 725-31, Jun 2012.

[157] P. Schmid, M. B. Wischnewsky, O. Sezer, R. Bohm, and K. Possinger, "Prediction of response to hormonal treatment in metastatic breast cancer," Oncology., vol. 63, pp. 309-16, 2002.

Received: August 06, 2013

Revised: September 06, 2013

Accepted: September 15, 2013

(C) Sutariya et al.; Licensee Bentham Open.

This is an open access article licensed under the terms of the Creative Commons Attribution Non-Commercial License (http://creativecommons.org/licenses/by$\mathrm{nc} / 3.0 /$ ), which permits unrestricted, non-commercial use, distribution and reproduction in any medium, provided the work is properly cited. 